\title{
Proposta de modelo para dimensionamento do pessoal de enfermagem em assistência domiciliária*
}

\author{
PROPOSAL FOR A MODEL FOR CALCULATING THE SIZE OF NURSING STAFF IN HOME CARE \\ PROPUESTA DE MODELO PARA LA PROVISIÓN DE PERSONAL DE ENFERMERÍA \\ ENASISTENCIADOMICILIARIA
}

Luiza Watanabe Dal Ben¹, Raquel Rapone Gaidzinski²

\section{RESUMO}

A assistência domiciliária $(\mathrm{AD})$ suscita questionamentos sobre a necessidade do número de horas diárias e dos dias de assistência de enfermagem por parte dos compradores da prestação de serviços à saúde em domicílio. Identificar os critérios adotados por gerentes e enfermeiras para o dimensionamento do pessoal de enfermagem. Os dados foram coletados por meio de entrevista semiestruturada e analisados segundo o método de análise de conteúdo. Identificaram-se três categorias: elegibilidade do paciente; tempo despendido na assistência e perfil de competência do profissional. As etapas consideradas foram: identificação da carga média diária de trabalho; determinação da proporção das categorias profissionais; jornada de trabalho dos profissionais de enfermagem e identificação do índice de segurança técnica para cobertura de ausências previstas e de não previstas. Com base nessas variáveis, propusemos um modelo para calcular o quadro de profissionais de enfermagem em $\mathrm{AD}$, agilizando o processo de tomada de decisão.

\section{DESCRITORES}

Serviços de enfermagem.

Recursos humanos de enfermagem.

Serviços de assistência

domiciliar.

\section{ABSTRACT \\ RESUMEN}

Home care brings up issues related to the number of daily hours and days of nursing service from users of these services. This study was carried out in order to identify the criteria adopted by managers and nurses for calculating the nursing staff needed in home care services. Data were collected through semi-structured interviews and analyzed according to the method of content analysis. Three categories were identified: patient's eligibility, time spent in care, and professional competency profile. The steps considered were: identification of daily average work load, choice of the proportion of professional categories, nursing workers' daily journey, and identification of the technical safety index for covering planned and unplanned absences. From these variables a model for calculating nursing personnel needed in home care is proposed in order to speed up the decision making process.

\section{KEY WORDS}

Nursing services.

Nursing staff.

Home care services.
La asistencia domiciliaria (AD) suscita interrogantes sobre la necesidad del número de horas diarias y de los días de asistencia de enfermería por parte de los compradores de prestación de servicios a la salud en domicilio. Tuvo por objetivo identificar los criterios adoptados por gerentes y enfermeras para el cálculo del personal de enfermería. Los datos fueron recolectados por medio de una entrevista semi-estructurada y analizados según el método de análisis de contenido. Se identificaron tres categorías: elegibilidad del paciente, tiempo invertido en la asistencia y perfil de competencia del profesional. Las etapas consideradas fueron: identificación de la carga promedio diario de trabajo; determinación de la proporción de las categorías profesionales; jornada de trabajo de los profesionales de enfermería e identificación del índice de seguridad técnica para la cobertura de ausencias previstas y de ausencias no previstas. A partir de esas variables propusimos un modelo para calcular el cuadro de profesionales de enfermería en $\mathrm{AD}$, agilizando el proceso de toma de decisiones.

\section{DESCRIPTORES}

Servicios de enfermería.

Personal de enfermería.

Servícios de atención de salud a domicílio.

\author{
Extraído da tese \\ "Dimensionamento \\ do pessoal de enfer- \\ magem em assis- \\ tência domiciliária: \\ percepção de geren- \\ tes e enfermeiras", \\ Escola de Enferma- \\ gem da Universidade \\ de São Paulo \\ (EEUSP), 2005 \\ 1 Enfermeira. Mestre \\ em Enfermagem pela \\ EEUSP. Presidente \\ do Grupo Dal Ben \\ Home Care \\ luiza@dalben.com.br \\ 2 Enfermeira. Professora \\ Livre-Docente do \\ Departamento de \\ Orientação Profis- \\ sional da EEUSP. \\ raqui@usp.br
}




\section{INTRODUÇÃO}

A assistência domiciliária (AD) é a provisão de serviços de saúde às pessoas de qualquer idade em seus lares, com os objetivos de: substituir a hospitalização repentina por necessidade aguda de cuidados, diminuir uma longa internação institucional e manter os indivíduos em seus domicílios e comunidade ${ }^{(1-2)}$.

Em nosso País, a partir de 1997, essa modalidade de assistência está regulamentada no Sistema Único de Saú$\mathrm{de}^{(3)}$ e o funcionamento dos serviços que prestam atenção domiciliar a partir de janeiro de 2006, pela Resolução da Diretoria Colegiada - RDC n ${ }^{\circ} 11$ da Agência Nacional de Vigilância Sanitária ${ }^{(4)}$

A Resolução COFEN no 267/2001 ${ }^{(5)}$ regulamenta as atividades de enfermagem em domicílio, e a Resolução COFEN no $270 / 2002^{(6)}$ regulamenta as empresas que prestam serviços de enfermagem domiciliar-home care, definindo a necessidade de ter enfermeira, como responsável técnico pela coordenação das atividades de enfermagem e uma en-fermeira responsável por turno de trabalho.

Esta modalidade assistencial exprime, significativamente, a autonomia e o caráter liberal do exercício profissional da enfermeira. As atividades de enfermagem estão previstas para os níveis de menor, média e alta complexidade, e contemplam as funções assistencial, administrativa, educativa e de pesquisa.

Quanto ao dimensionamento de pessoal assistência domiciliária de enfermagem, a Resolução COFEN no 293/ $2004^{(7)}$ fixa e estabelece parâmetros para o dimen-sionamento do quadro de profissionais de enfermagem nas instituições de saúde e assemelhados, estabelece o quanti-qualitativo mínimo nos diferentes níveis de formação dos profissionais de enfermagem para a cobertura assistencial nas instituições de saúde.

Embora se possa entender os assemelhados, essa Resolução não contempla o dimensionamento do pessoal de enfermagem nos ambulatórios e em AD.

Conforme pesquisadoras brasileiras ${ }^{(8)}$ a temática dimensionamento de pessoal de enfermagem tem se constituído ao longo dos anos, foco de atenção das enfermeiras, bem como dos administradores dos serviços de saúde, por interferir, diretamente, na eficácia e no custo da assistência à saúde.

Afirma-se que diante do desconhecimento de parâmetros para o planejamento e avaliação do quantitativo de recursos humanos de enfermagem, o dimensionamento de pessoal de enfermagem tem sido realizado de forma empírica, baseado na experiência e no julgamento intuitivo das enfermeiras, ou por meio de utilização de equações matemáticas disponíveis na literatura, sem que haja compreensão do significado e do comportamento das variáveis envolvidas no processo de dimensionar o pessoal de enfermagem ${ }^{(9)}$.

A AD suscita o questionamento sobre a real necessidade do número de horas diárias e dos dias de assistência de enfermagem por parte dos compradores da prestação de serviços à saúde em domicílio que, com vistas à redução dos custos, procuram limitar esse quantitativo. $\mathrm{O}$ bom desempenho da equipe de enfermagem reflete-se diretamente na satisfação dos pacientes, de suas famílias e da equipe interdisciplinar. Este desempenho está diretamente relacionado com a adequação do tempo de permanência do profissional de enfermagem no domicílio, de acordo com as necessidades do paciente, seu perfil pessoal e profissional, como também, com à viabilidade dos custos da assistência e à supervisão mantida pela enfermeira ${ }^{(10-11)}$.

Acredita-se que a sistematização do conhecimento das enfermeiras, bem como, a utilização de intrumentos que permitam quantificar o tempo despendido nas intervenções de enfermagem aos pacientes em AD poderá instrumentalizar essas profissionais nas negociações referente ao quadro de profissionais de enfermagem que permita uma assistência orientada na busca da qualidade do cuidado de enfermagem.

A presente pesquisa objetivou identificar os critérios utilizados por gerentes e enfermeiras que atuam na área, para dimensionar o pessoal de enfermagem e, propor um modelo que norteie a sistematização do dimensionamento do pessoal de enfermagem em assistência domiciliária.

\section{TRAJETÓRIA METODOLÓGICA}

O estudo é exploratório descritivo com abordagem qualitativa, os dados foram coletados por meio de entrevistas semi-estruturadas, no período de abril a julho de 2004, gravadas com a permissão dos entrevistados e posteriormente, transcritas. Os entrevistados assinaram o Termo de Consentimento Livre e Esclarecido, e receberam uma cópia da aprovação do projeto desta pesquisa pelo Comitê de Ética em Pesquisa de Escola Enfermagem da Universidade de São Paulo.

As questões norteadoras foram relativas à descrição da indicação de um paciente para $\mathrm{AD}$ e ao planejamento do dimensionamento do pessoal de enfermagem.

O teste-piloto foi realizado com duas enfermeiras com experiência de cinco anos na área de atendimento e internação domiciliária para verificar a compreensão das perguntas norteadoras. 
Participaram do estudo 48 profissionais, pertencentes a 4 instituições do setor público e 20 do setor privado, da cidade de São Paulo. Destes, 24 eram gerentes dos serviços de AD e 24 enfermeiras eram coordenadoras das equipes de enfermagem.

Quanto à formação dos entrevistados que exerciam a função de gerentes, dez $(41,6 \%)$ eram médicos, seis $(25 \%)$ enfermeiras, dois $(8,2 \%)$ analistas contábeis, uma $(4,2 \%)$ nutricionista, uma (4,2\%) psicóloga, um (4,2\%) engenheiro, uma (4,2\%) bióloga, um $(4,2 \%)$ administrador e um $(4,2 \%)$ com curso superior incompleto. A média do tempo de experiência em $\mathrm{AD}$ das enfermeiras na função de gerentes foi de 11,8 anos.

O acesso aos profissionais do setor privado foi obtido por intermédio de solicitação às empresas vinculadas ou registradas na Associação Brasileira de Empresas de Medicina Domiciliar (ABEMID), na Associação Brasileira de Home Health Care (ABRAHHCARE), na Sociedade Brasileira de Enfermagem em Home Care (SOBEHC), no Núcleo Nacional de Empresas de Assistência Domiciliar (NEAD) e nas empresas inscritas no Conselho Regional de Enfermagem do Estado de São Paulo (COREN - SP). Houve retorno da ABEMID, NEAD e do COREN-SP.

A solicitação para realização das entrevistas, juntamente com o projeto de pesquisa e aprovação do Comitê de Ética em Pesquisa, foi enviada para 39 instituições, houve retorno de 26 , sendo que duas informaram que haviam encerrado suas atividades, totalizando 24 organizações.

As entrevistas foram agendadas e realizadas pela pesquisadora, em local reservado, na sede das organizações com exceção de dois profissionais que optaram por outro local de trabalho e foram gravadas com a permissão dos entrevistados para serem posteriormente transcritas.

A técnica de análise de conteúdo ${ }^{(12)}$ foi aplicada por ser considerada uma forma para afastar o perigo de uma compreensão espontânea, intuitiva, livre e reduzir o risco de gerar um material de conteúdo superficial, sobretudo, uma análise linear descritiva, ou ainda, fazer interpretações originadas de interferências destituídas de fundamentação lógica expondo, assim, a tendenciosidade pessoal da pesquisadora. O material obtido dos depoentes foi analisado em três fases: pré-análise, exploração do material e tratamento dos resultados obtidos e interpretação.

\section{RESULTADOS}

A análise interpretativa dos 48 discursos possibilitou a construção de três categorias que elucidam o contexto e os critérios considerados para dimensionar o pessoal de enfermagem pelos gerentes $(\mathrm{G})$ e enfermeiras (E) de serviços públicos $(\mathrm{Pb})$ e privados $(\mathrm{Pv})$ na $\mathrm{AD}$ : elegibilidade do paciente; tempo despendido na assistência e perfil de competência profissional.
Elegibilidade do paciente: A indicação do paciente para $\mathrm{AD}$ é da competência do médico, sendo apontada como uma condição em todos os depoimentos dos entrevistados dos setores público e privado. A viabilidade é avaliada pela assistente social e pela enfermeira.

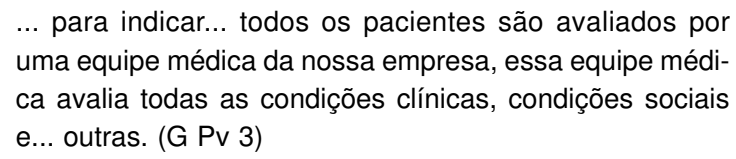

A assistente social vai a casa, para avaliar as condições do ambiente do paciente. Após essa visita do serviço social, a família vem até a gente. E a partir dali, eu faço uma entrevista com ele também. Ele vem até o hospital, eu faço uma entrevista com a família do paciente. (E Pb 2)

A estabilidade clínica do paciente, condições de moradia, a região de seu domicílio, presença obrigatória do cuidador familiar e redução do custo da assistência hospitalar foram os outros indicadores para a elegibilidade do paciente. Os entrevistados, do setor público e privado, destacaram a estabilidade clínica como quesito indispensável, para que o paciente seja atendido em seu domicílio. Os serviços do setor público associam a estabilidade clínica à idade dos pacientes, apontando como um dos critérios de eleição a faixa etária, acima de 60 anos.

Primeiramente, a estabilidade clínica, eu levo para casa o paciente que está clinicamente estável, começamos por aí, ... (E Pv 12)

$$
\begin{aligned}
& \text {... o paciente, tem que ter uma casa em condições mínimas } \\
& \text { para sobreviver... (E } \mathrm{Pb} 1)
\end{aligned}
$$

... importante é que tenha um cuidador, ter uma família que apóie e ter um cuidador responsável, porque nós não elegemos paciente que não tenha um cuidador que more junto, seja um parente, seja um amigo, então, ele tem que ter esse cuidador. $\mathrm{E} \mathrm{Pb} 1$

Observa-se que a $\mathrm{AD}$ constitui-se em um repasse de custos à família, como: medicamentos, nutrição e lavanderia que, na estrutura hospitalar, são absorvidos pelo hospital, além da transferência do cuidado como uma responsabilidade familiar.
... a família é quem arca com os custos das despesas, de medicação, curativos. Nós cuidamos, ensinamos, e de- pois passamos essa responsabilidade, para que eles pos- sam continuar cuidando. (E Pv 20)

Tempo despendido na AD: Os critérios para dimensionamento do pessoal de enfermagem, relatados pelos entrevistados, são ancorados no tipo e na quantidade de intervenções presentes no paciente, indicando o grau de dependência do paciente em relação aos cuidados de enfermagem, para isso, utilizam-se da prescrição médica.

... quantas vezes, o paciente é aspirado por dia ... precisa ter um auxiliar lá, quantas medicações endovenosas ... quais 
são os horários então, é em relação a esses problemas ativos, que nós computamos as horas ... calculamos a quantidade de horas de enfermagem ... precisa de um treinamento do cuidador. Então, a gente identifica mais ou menos o tempo para trabalhar esses problemas ativos que se dimensionam, esse número de horas de enfermagem. (G Pv 9)

Os tipos de modalidades praticados em AD relacionam-se diretamente ao quantitativo do pessoal de enfermagem. $\mathrm{O}$ atendimento domiciliário compreende a realização de visitas pontuais e a execução de intervenções de enfermagem. A internação domiciliária exige a permanência do profissional de enfermagem no mínimo de 6 horas até 24 horas no domicílio do paciente.

A classificação do grau de dependência do paciente está diretamente relacionada ao quantitativo de horas de assistência de enfermagem necessárias ao mesmo, como se constata pelo depoimento do gerente G Pv 1 .

Então, nós classificamos hoje, de acordo com o número de horas de enfermagem, que é uma prática mais ou menos igual, para todos os home care. Então, temos desde o nível de 6 horas de enfermagem até 24 horas. (G Pv 1)

Para o dimensionamento de enfermeiras, adota-se outra forma de cálculo, estipulam-se dez pacientes adultos para cada profissional, reduzindo para sete se for de pediatria.

Com as enfermeiras, eu tenho uma outra dinâmica ... na equipe da pediatria, eu trabalho com a média de sete pacientes por enfermeira e no Home Care adulto, eu trabalho com a média de dez pacientes por enfermeira, variando um pouco. (E Pv 3)

A escala dos profissionais de enfermagem está diretamente relacionada, de acordo com a disponibilidade dos profissionais, o que difere, nos serviços privados, diametralmente com a escala realizada em instituições hospitalares submetidas ao regime da Consolidação de Leis Trabalhistas (CLT).

Então, a partir do momento que eu tenho uma solicitação, esse pedido, muitas vezes, ele vai demandar a montagem de uma equipe de internação, que vai requerer, normalmente, quatro auxiliares e um quinto como folguista. Então, tem uma enfermeira supervisora em cada região de São Paulo. $E$ essa enfermeira ... os auxiliares autônomos, eles se reportam a essa enfermeira supervisora da região. Assim, como a gente também trabalha com uma equipe que tem condições de fazer atendimento. com horas a mais, 12 horas de enfermagem, 8 horas de enfermagem, 6 horas. $\mathrm{E}$ a gente também tem aqueles casos dos auxiliares que só têm condições de fazer, por exemplo, atendimento pontual. Então, a gente acaba trabalhando com uma carteira bem ampla. Tem auxiliar de enfermagem que tem condições de fazer um atendimento de duas horas, três horas, até aquele que está full time. Ele pode, ele tem condições de fazer plantão, tanto no dia par ou ímpar 12horas. (E Pv 19)

Para as instituições públicas especializadas (Psiquiatria e Oncologia) ou unidade especializada em cuidados paliativos, o tempo despendido na $\mathrm{AD}$ está diretamente relacionado com a doença apresentada pelo paciente e sua dificuldade de acesso ao hospital. O quadro de pessoal de enfermagem é fixo.

A indicação de paciente ao nosso serviço de atendimento domiciliar, para os cuidados paliativos, ele tem que estar fora de chance terapêutica, não estar respondendo ao controle dos sintomas.] (G $\mathrm{Pb}$ 2)

A principal indicação é: o paciente ter o transtorno psiquiátrico, que precisa de assistência em saúde mental, mas que não esteja conseguindo chegar até o Instituto de Psiquiatria, ou seja, a questão é a dificuldade de acesso, ele não consegue chegar até o ambulatório ... pela própria condição psiquiátrica. (G Pb 4)

Perfil de competência profissional: A filosofia das organizações influencia diretamente no quantitativo e qualitativo de pessoal de enfermagem em AD, seja pela demanda em qualidade da prestação de serviços, como suporte de desenvolvimento do pessoal em AD no trabalho multidisciplinar.

A realidade vivenciada pelo setor público limita o quantitativo e qualitativo do pessoal de enfermagem. $O$ gerente $G$ $\mathrm{Pb} 3$ em seu depoimento expõe que a enfermeira não está autorizada a realizar curativos pela demanda de outras atividades que não permitem a atividade direta assistencial.

... nós damos uma assistência que não é a ideal, por causa de problemas de recursos do hospital, então nós só temos dois médicos, uma enfermeira que não está autorizada a fazer curativo, para atender esses pacientes como conseqüência da doença de base, eles têm escaras de decúbito. ( $\mathrm{G} \mathrm{Pb} 3$ )

O conhecimento técnico-científico do profissional também interfere no dimensionamento do pessoal em AD.

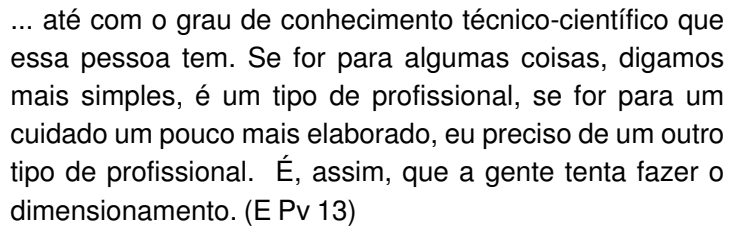
cuidado um pouco mais elaborado, eu preciso de um outro tipo de profissional. É, assim, que a gente tenta fazer o dimensionamento. (E Pv 13)

A legislação do exercício profissional da enfermagem possibilita que os técnicos de enfermagem realizem procedimentos invasivos; no entanto, a realidade vivenciada pelas empresas carece em quantidade e experiência desse profissional.

\begin{abstract}
A gente tem uma meta de até dezembro de 2005, é ter todos os nossos funcionários na área de enfermagem: técnicos. Mas, ainda, é um futuro. Atualmente, a gente tem técnicos e tem auxiliar....nós temos excelentes auxiliares de enfermagem com uma vivência muito grande e são super valorizados dentro do serviço. (G Pv 11)
\end{abstract}

O papel da educação continuada apresenta características peculiares a essa modalidade de atendimento, precisando de um monitoramento rigoroso do comportamento, das habilidades e atitudes dos profissionais, cabendo-lhes a orientação, o realocamento em cada domicílio. 
... a educação continuada faz um trabalho muito interessante, acompanha as situações dos cooperados, faz treinamento, de postura, de comportamento, de técnica. Esses profissionais estão dentro do modelo hospitalar, não sabem trabalhar dentro do contexto familiar e domiciliar... o envolvimento pessoal. Ele não sabe trabalhar com a interferência da família, ele não sabe como reagir frente a um estresse da família ou a uma resistência do paciente. Essas situações para o profissional são novas. ( $\mathrm{P}$ v 9)

A quantidade e a qualidade das intervenções presentes no paciente demandam conhecimento científico e habilidades do profissional, influenciando diretamente no dimensionamento do pessoal de enfermagem.

Vários autores ${ }^{(13-15)}$ afirmam que a formação profissional específica para atender aos pacientes e suas famílias em suas casas é deficiente.

A maioria dos profissionais de enfermagem que presta serviços em $\mathrm{AD}$ no setor privado, trabalha em regime de cooperativismo $^{(16)}$.

\section{Proposta de modelo para o dimensionamento do pessoal em assistência domiciliária}

Os resultados da análise dos depoimentos dos gerentes e enfermeiras, somados à vivência da autora na assistência a pacientes que estão no domicílio, permitem sistematizar o interrelacionamento das variáveis que influenciam o dimensionamento de pessoal da equipe de enfermagem que presta $\mathrm{AD}$.

Nessa perspectiva, o método para dimensionar o pessoal de enfermagem proposto ${ }^{(8)}$ pode ser adaptado a essa modalidade de assistência, considerando-se as seguintes etapas: identificação da carga média de trabalho diária na assistência domiciliária (AD), determinação da proporção da categoria profissional da enfermagem e jornada de trabalho da equipe de enfermagem.

Para pesquisadoras brasileiras ${ }^{(8)}$, a carga de trabalho consiste no produto da quantidade média diária de pacientes/clientes assistidos, conforme o grau de dependência do paciente à equipe de enfermagem ou do tipo de intervenção pelo tempo médio de assistência de enfermagem utilizado, por cliente, de acordo com o grau de dependência ou intervenções realizadas.

Ou seja: $C=\sum_{j}(n j . h j)$ onde:

$C=$ carga de trabalho;

$n j=$ quantidade média diária de pacientes/clientes assistidos, conforme o grau de dependência ou o tipo de intervenções;

$h j$ = tempo médio diário de cuidado, conforme o grau de dependência ou o tipo de intervenção;

$j=$ qualquer grau de dependência ou tipo de intervenção.

A quantidade de pacientes/clientes (nj) é determinada pela média diária de pacientes/clientes assistidos, de acordo com o grau de dependência do paciente à equipe de enfermagem ou da quantidade de intervenções realizadas.
O tempo médio despendido no cuidado, conforme o grau de dependência do paciente e segundo os instrumentos de classificação é considerado como Dependência Total: 24 horas de assistência/dia/paciente; Dependência Parcial: 12 horas de assistência / dia / paciente; Dependência Moderada: 6 horas de assistência / dia / paciente.

Para a modalidade atendimento domiciliário, o tempo será o somatório das intervenções a serem realizadas.

No que diz respeito à determinação do percentual de cada categoria da equipe de enfermagem $(\mathrm{Pk})$, ressalta-se que esta varia, de acordo com o significado atribuído à assistência de enfermagem e com a disponibilidade de profissionais no mercado de trabalho ${ }^{(17)}$. Portanto, após a identificação da carga de trabalho é necessário distinguir qual a proporção da carga de trabalho que caberá à enfermeira e técnico/auxiliar de enfermagem.

A jornada de trabalho dos profissionais de enfermagem, é, geralmente, $12 \mathrm{~h}$ por $36 \mathrm{~h}$, como autônomos vinculados às cooperativas de trabalho.

De acordo com a mesma autora ${ }^{(17)}$, desde os primeiros estudos sobre o trabalho, considera-se que os trabalhadores não são produtivos, igualmente, em todo o tempo do turno de trabalho, por realizarem uma série de atividades não diretamente relacionadas às suas tarefas profissionais, como: atendimento de suas necessidades fisiológicas; períodos de descanso; trocas de informações não ligadas ao trabalho; deslocamentos; comemorações e outras.

Dessa forma, quando se tratar de atendimento domiciliário, sugere-se que sejam consideradas as perdas de produtividade dos trabalhadores de enfermagem, mediante a redução das horas disponíveis do trabalhador em seus turnos de trabalho, de maneira que o tempo efetivo de trabalho seja assim, mensurado:

onde:

$$
t_{\text {efetivo }}=t_{k} \cdot p_{k}
$$

$t k=$ jornada de trabalho da categoria profissional $p k=$ proporção do tempo produtivo da categoria profissional.

A vivência vem apontando que a localização dos domicílios dos pacientes a serem atendidos interfere no quantitativo do pessoal de enfermagem, sobretudo quando a carga horária diária é menor do que 6 horas. Estas atividades são apontadas em estudos ${ }^{(10-11)}$, como isoladas.

\section{Índice de Segurança Técnica}

A determinação de um índice de segurança técnica consiste em um acréscimo no quantitativo de pessoal de enfermagem por categoria profissional, para a cobertura das ausências ao serviço.

Há autor ${ }^{(17)}$ que entende como ausências previstas os dias relativos às folgas (descanso semanal remunerado e 
feriados) e às férias e como ausências não previstas os dias referentes às faltas, licenças e suspensões.

A denominação Índice de Segurança Técnica (IST), tem sido utilizada para designar os percentuais para a cobertura desses tipos de ausências.

A Resolução COFEN n. 293/96(7), estabeleceu que ao quantitativo de profissionais necessários para a prestação da assistência de enfermagem deverá ser acrescido um IST não inferior a $15 \%$.

Os percentuais referentes a cada tipo de ausências podem ser calculados a partir das equações propostas ${ }^{(17)}$.

Ausências previstas por folga semanal (E\%): As cargas de trabalho semanal, mais freqüentemente adotadas nas instituições de saúde do país são: o sistema de 36 horas de trabalho por semana, com uma folga semanal e o sistema de 30 horas de trabalho por semana, com duas folgas semanais.

O valor percentual do acréscimo de pessoal devido a variável folgas semanais por trabalhador E\% pode ser calculado através da equação:

onde:

$$
E \%=\frac{e}{d-e} \cdot 100
$$

$E \%=$ percentual de folgas;

$e=$ número de dias de folga, por semana, dos trabalhadores da enfermagem;

$d=$ número de dias trabalhados na unidade.

Ausências previstas por feriado (F\%): Nos serviços ininterruptos de enfermagem todo o quadro de pessoal tem direito a tantos dias de folgas, quanto forem os feriados não coincidentes com os domingos. O número de folgas varia, de acordo com o ano, em função da quantidade dos feriados não coincidentes com o domingo.

Calcula-se os valores percentuais limites dos acréscimos devidos aos dias feriados F\%, através da aplicação da equação:

$$
F \%=\frac{f}{D-f} \cdot 100
$$

onde:

$F \%=$ percentual de dias feriados;

$f=$ dias feriados no ano;

$D=$ dias do ano, 365 dias.

Ausências previstas por férias (Vk\%): O valor máximo para férias, conforme a legislação, é de 30dias/ano por trabalhador de qualquer categoria profissional, que pode ser expresso com a equaçâo a seguir:

onde:

$$
V_{k} \%=\frac{v k}{D-v k} \cdot 100
$$

$V k \%=$ percentual de férias, segundo a categoria profissional ${ }_{k}$ (enfermeiro, técnico/auxiliar);

$v k=$ média de dias de férias da categoria profissional ${ }_{k}$;

$D=$ dias do ano, 365 dias.
Ausências não previstas (Ak\%): A variável ausências não previstas é resultante da soma de uma série de tipos de ausência, tais como: faltas abonadas e não abonadas; licença médica; licença maternidade; licença prêmio; licença por acidente de trabalho; licença INSS; outras licenças (casamento, nojo, paternidade, etc.), cujos valores diferem de uma categoria profissional para outra.

Como a ausência não prevista é composta pela soma das diversas ausências já relatadas anteriormente, podemos representá-la com a seguinte equação:

$$
A_{k} \%=\left(\frac{\sum_{i} a_{k, i}}{D-\sum_{i} a_{k, i}}\right) \cdot 100
$$

Onde:

$A k \%=$ percentual de ausências não previstas, segundo a categoria profissional k (enfermeiro, técnico /auxiliar)

$\sum_{i} a_{k, i}=$ somatória dos dias médios de ausências não previstas, segundo

os tipos de ausências (faltas, licenças e suspensões) por categoria profissional ${ }_{\mathrm{k}}$ (enfermeiro, técnico e auxiliar); $D=$ dias do ano, 365 dias.

Diante dos resultados encontrados referentes às ausências previstas e não previstas calcula-se o IST, para o acréscimo de pessoal de enfermagem, conforme demonstrado a seguir:

$$
I S T_{k} \%=\left\{\left[\left(1+\frac{E \%}{100}\right) \cdot\left(1+\frac{F \%}{100}\right) \cdot\left(1+\frac{V_{k}}{100}\right) \cdot\left(1+\frac{A_{k}}{100}\right)\right]-1\right\} \cdot 100
$$

Praticamente em todas as organizações privadas de AD, a contratação do pessoal de enfermagem é efetuada por cooperativas de trabalho, por isso, o IST para cobertura das ausências previstas e não previstas, não deve ser contemplado neste segmento.

Contudo, a vivência da pesquisadora demonstra que não havendo a preocupação com a cobertura de ausências, há necessidade de profissionais qualificados e aptos para atuarem nesse modelo de assistência. O depoimento dos gerentes e das enfermeiras deste estudo, mostra a importância da educação continuada em suas organizações. Por outro lado, a substituição dos profissionais que pertencem às cooperativas de trabalho, é de responsabilidade das empresas que devem apresentar prontamente um outro profissional autônomo para as organizações de AD. Por se tratar de trabalho no cenário domiciliar do paciente, a questão não é simples. Sempre deve haver aceitação por parte do paciente e de sua família, como também do profissional.

Sintetizando pode-se expressar genericamente o dimensionamento de pessoal de enfermagem por meio da equação proposta por pesquisadoras brasileiras ${ }^{(8)}$, convenientemente simplificada para se adaptar à realidade da $\mathrm{AD}$ : 
onde: $\quad Q=\frac{\frac{P_{k j}}{100} \cdot\left(n_{j} \cdot h_{j}\right)}{t_{k} \cdot p_{k}} \cdot I S T_{k}$

$Q=$ quantidade total de pessoal de enfermagem;

$P k j=$ percentual do trabalho dedicado ao tipo de cuidado ${ }_{j}$ pela categoria profissional ;

$n j=$ média diária de pacientes que necessitam do cuidado ;

$h j=$ média das horas de assistência de enfermagem por paciente que necessita do cuidado

\section{CONSIDERAÇÕES FINAIS}

O modelo de $\mathrm{AD}$ adotado pelo serviço público difere do setor privado por não oferecer a internação domiciliária, tornando-os distintos nesse aspecto. $\mathrm{O}$ atendimento domiciliário e o monitoramento de doenças crônicas, em ambos os serviços são realizados por uma equipe multidisciplinar.

A percepção dos gerentes e das enfermeiras de serviços públicos e privados em $\mathrm{AD}$, no que se refere ao dimensionamento do pessoal de enfermagem, não diferiu em sua essência, pois ambos adotam os mesmos critérios para calcu-

\section{REFERÊNCIAS}

1. Hierschfeld MJ, Oguisso T. Visão panorâmica da saúde no mundo e a inserção do home care. Rev Bras Enferm. 2002;55(4):452-9.

2. World Health Organization (WHO). Home care issues at the approach of the $21^{\text {st }}$. Century from a World Health Organization perspective: a literature review. Geneva: WHO; 1999. pt. 1, p. 1-39.

3. Brasil. Portaria n. 2416, de 23 de março de 1998. Estabelece requisitos para credenciamento de hospitais e critérios para realização de internação domiciliar no SUS. Diário Oficial da União, Brasília, 26 mar. 1998. Seção 1, p. 106.

4. Agência Nacional de Vigilância Sanitária (ANVISA). Resolução Diretoria Colegiada n. 11, de 26 de janeiro de 2006. Dispõe sobre o regulamento técnico para o funcionamento de serviços que prestam atenção domiciliar. Diário Oficial da União, Brasília 30 jan. 2006. Seção 1, p. 78.

5. Conselho Federal de Enfermagem (COFEn). Resolução n. 267, de 5 de outubro de 2001. Aprova atividades de enfermagem em domicílio home care [legislação na Internet]. Rio de Janeiro; 2001.[citado 2005 jun. 5]. Disponível em: http://www. bve.org.br/ portal materias.asp? ArticleID $=1251 \&$ SectionID $=194 \& \mathrm{Sub}$ SectionID $=194 \&$ SectionParentID $=189$

6. Conselho Federal de Enfermagem (COFEn). Resolução n. 270, de 18 de abril de 2002. Aprova a regulamentação das empresas que prestam serviços de Enfermagem Domiciliar - Home Care. [legislação na Internet]. Foz de Iguaçu; 2002. Disponível em: http://www. portalcofen.com.br [citado 2005 jun. 5].

7. Conselho Federal de Enfermagem(COFEn). Resolução n. 293, de 21 de setembro de 2004. Fixa e estabelece parâmetros para o dimensionamento do quadro de profissionais de enfermagem nas Unidades Assistenciais das Instituições de Saúde e Assemelhados. [legisção na Internet]. Rio de Janeiro; 2004. Disponível em: http://www.corensp.org.br/resoluções/ Resolução293.htm [citado jun. 2005]. lar o número de profissionais. As categorias consideradas após a análise interpretativa dos 48 discursos foram: elegibilidade do paciente, tempo despendido na assistência e perfil de competência profissional.

O dimensionamento de pessoal de enfermagem, em particular para a $\mathrm{AD}$ constitui ainda um grande desafio para o gerenciamento da assistência. A vivência relatada pelos entrevistados, e a experiência vivida por uma das autoras desta pesquisa, permitiram indicar um caminho na construção de um modelo de dimensionamento de pessoal de enfermagem em AD, fundamentado no método desenvolvido por Gaidzinski ${ }^{(17)}$, considerando as etapas: identificação da carga média de trabalho diária na assistência domiciliária; determinação da proporção da categoria profissional da enfermagem; jornada de trabalho da equipe de enfermagem e índice de Segurança Técnica.

Os resultados apresentados neste estudo, poderão facilitar um adequado planejamento do quadro de pessoal de enfermagem, agilizando a comunicação entre os administradores, enfermeiras, gerentes de empresas de AD, planos de saúde do setor privado e profissionais do setor público.

8. Gaidzinski RR, Fugulin FMT, Castilho V. Dimensionamento de pessoal de enfermagem em instituições de saúde. In: Kurckgant P, coordenadora. Gerenciamento de enfermagem. São Paulo: Guanabara Koogan; 2005. p. 125-37.

9. Gaidzinski RR. O dimensionamento do pessoal de enfermagem segundo a percepção de enfermeiras que vivenciam essa prática [tese]. São Paulo: Escola de Enfermagem, Universidade de São Paulo; 1994.

10. Dal Ben LW. Instrumento para dimensionar horas diárias de assistência de enfermagem residencial [dissertação]. São Paulo: Escola de Enfermagem, Universidade de São Paulo; 2000.

11. Dal Ben LW, Sousa RMC. Adaptação de instrumento para dimensionar horas diárias de assistência de enfermagem residencial. Rev Esc Enferm USP. 2004;38(1):80-9.

12. Bardin L. Análise de conteúdo. Lisboa: Edições 70; 1977.

13. Angerami ELS, Gomes DLS. Análise da formação do enfermeiro para a assistência de enfermagem no domicílio. Rev Lat Am Enferm. 1996;4(2):5-22.

14. Andrade L, Jacob Filho W. Perfil dos cuidadores de pacientes portadores de demência atendidos por programa de assistência domiciliar. Rev Bras Home Care. 2003;7(96):12-3.

15. Cruz LP. Assistência domiciliar: um estudo sobre a formação de profissionais e a prestação de serviços no estado de São Paulo [dissertação]. São Paulo: Fundação Getúlio Vargas; 1994.

16. Bráz MG. Relações de trabalho em uma empresa de home care. Rev Bras Home Care. 2001;6(72):10-2.

17. Gaidzinski RR. Dimensionamento de pessoal de enfermagem em instituições hospitalares [tese livre-docência]. São Paulo: Escola de Enfermagem, Universidade de São Paulo; 1998. 